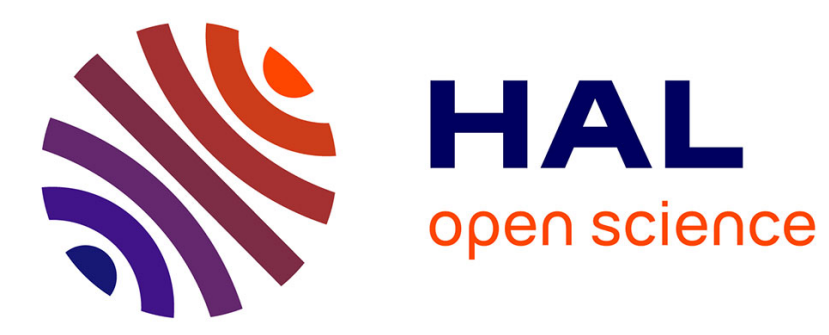

\title{
Compliant Surfaces under Shear: Elastohydrodynamic Lift Force
}

\author{
Pierre Vialar, Pascal Merzeau, Suzanne Giasson, Carlos Drummond
}

\section{To cite this version:}

Pierre Vialar, Pascal Merzeau, Suzanne Giasson, Carlos Drummond. Compliant Surfaces under Shear: Elastohydrodynamic Lift Force. Langmuir, 2019, 35 (48), pp.15605-15613. 10.1021/acs.langmuir.9b02019 . hal-02383852

\section{HAL Id: hal-02383852}

\section{https://hal.science/hal-02383852}

Submitted on 25 Nov 2020

HAL is a multi-disciplinary open access archive for the deposit and dissemination of scientific research documents, whether they are published or not. The documents may come from teaching and research institutions in France or abroad, or from public or private research centers.
L'archive ouverte pluridisciplinaire HAL, est destinée au dépôt et à la diffusion de documents scientifiques de niveau recherche, publiés ou non, émanant des établissements d'enseignement et de recherche français ou étrangers, des laboratoires publics ou privés. 


\section{Compliant Surfaces under Shear:}

\section{Elastohydrodynamic Lift Force}

Pierre Vialar, ${ }^{1,2}$ Pascal Merzeau ${ }^{1,2}$, Suzanne Giasson ${ }^{3 *}$ and Carlos Drummond ${ }^{1,2^{*}}$

1 CNRS, Centre de Recherche Paul Pascal (CRPP), UMR 5031, F-33600 Pessac, France

2 Université Bordeaux, CRPP, F-33600 Pessac, France

3 Department of Chemistry and Faculty of Pharmacy, Université de Montréal, C.P. 6128, succursale Centre-Ville, Montréal, QC, Canada, H3C 3J7

\section{Corresponding Author}

* drummond@crpp-bordeaux.cnrs.fr, suzanne.giasson@umontreal.ca

ABSTRACT. In this work we have investigated the behavior under shear and compression of mica surfaces coated with poly (N-isopropylacrylamide) cationic microgels. We have observed the emergence of velocity dependent, shear-induced normal forces, which can be large enough to entrain a fluid film that separates the surfaces out of contact, driving the dynamic system from conditions of boundary to hydrodynamic lubrication. By implementing a feedback-loop control on the surface separation, we were able to quantify the magnitude of the lift force as a function of the surface separation and driving speed. Our results illustrate how elastohydrodynamic effects can play an important role in the lubrication of compliant surfaces, providing pathways for control of friction and wear. 


\section{INTRODUCTION}

Surface Forces govern the outcome of a significant number of physical processes; colloidal stability, adhesion, wettability, boundary lubrication or capillarity can all be better described if the different surface forces involved are correctly taken into account. Modern experimental studies of interaction between solids dates back to the 1920s, when Tomlinson investigated the interaction between orthogonal metal filaments. ${ }^{1}$ Later, efforts headed by Overbeek and Derjaguin led to the development of reliable techniques for measuring the distance-dependent force between surfaces of quartz or glass. ${ }^{2,3}$ These earlier technical developments were constrained by the difficulty of manufacturing sufficiently smooth and clean substrates, a significant problem at small surface separations. As a solution to this problem, the use of mica as a substrate for surface force experiments was suggested by Bailey and Courtney-Pratt. ${ }^{4}$ Mica can be cleaved to produce macroscopic, molecularly smooth surfaces, ideal for measuring surface forces at small separations and evaluating theoretical developments. Based on the use of this substrate, a remarkable body of work was accomplished, leading to the development of the Surface Forces Apparatus, SFA, by Tabor, Winterton and Israelachvili. ${ }^{4,5}$ The technique was later developed to allow measurement in different liquid and gaseous environments. ${ }^{6}$ It was initially proposed to use multiple beam interferometry (MBI) to determine the actual separation between the surfaces $D$ with subnanometric resolution. Israelachvili developed simple analytical expressions to calculate the thickness and refractive index of a film confined between mica surfaces. ${ }^{7}$ This information is central for the quantitative description of surface forces, placing the SFA at the leading-edge in techniques of surface force measurement.

Different strategies have been developed for measuring normal interaction forces in the SFA. In the quasi-static approach originally used by Israelachvili and coworkers, one of the surfaces is attached to a loading spring $\left(K_{N}\right.$, Fig. 1$)$. When the system is at mechanical equilibrium, surface forces (if present) are balanced by the elastic force of the spring. To measure the static force of interaction between the surfaces, $F_{N}$, the separation between the other surface and the distal spring end ( $T$, in Fig. 1$)$ is changed in steps. After this movement, any difference between the variation of $T$ and the change in surface separation determined by MBI, $\Delta D$, signals a deflection of the normal spring, from which the change in the normal interaction force can be readily calculated if $K_{N}$ is known. This procedure might be limited by mechanical instabilities if the absolute value of the variation of the force with separation, $d F_{N} / d D$, is larger than $K_{N}$. Derjaguin and co-workers proposed the use of a feedback technique, applying and additional force (e.g. magnetic) to maintain the spring undeflected at all times ${ }^{3}$ eliminating the mechanical instability. Several implementations of this idea have been reported. ${ }^{8,9,10}$

Static surface forces have been rationalized in terms of different contributions (e.g. electrostatic, dispersive, hydrophobic or steric). But equilibrium forces are only one aspect of the interactions governing the properties of many systems of interest. Under out-of-equilibrium conditions, or in presence of changing conditions, the scenario may be more involved. For instance, considerable efforts have been carried out to study of the interaction between contacting surfaces in relative motion (tribology). In a typical nanotribology experiment with the SFA, the surfaces are brought to a certain separation $D$. Then, a lateral displacement between the surfaces is imposed at a given velocity $V$, and the tangential force induced by this displacement is measured. In these dynamic studies the response of the system to a changing environment can influence the boundaries (e.g., the value of $D$ or the shape of the surfaces) in ways that may depend on the specifics of the 
experimental setup. These changes complicate the process of separating intrinsic aspects of the system under study from the measuring device, and make it more difficult to compare results from different laboratories using different techniques or experimental setups. In addition, theoretical developments are often built considering unchanging contact geometry, or assuming that the surface separation remains unaffected sufficiently far from the contact zone, effectively assuming that the surfaces are fixed in an infinitely stiff housing. This may facilitate the task of modelling, but is often not accurately reproduced in practical circumstances. For instance, in many biotribological problems the rubbing surfaces are attached to compliant or deformable supports, with multiple degrees of freedom. In the case of force measuring devices, a spring of finite calibrated stiffness is commonly used to apply the external load $L$, by balancing the elastic spring force with the surface forces. If there is a temporal evolution of these forces, the spring deflection (and the relative position of the surfaces) will change accordingly.

The SFA allows a direct determination of the geometry of the contact in real-time based on MBI. Few decades ago, it was a demanding and time consuming task to obtain accurate measurements. Nowadays, fast and sensitive cameras, computers, and fast algorithms allow precise determination of the geometry in real time. The fast determination of the contact geometry, unique to the SFA technique, together with faster an accurate piezoelectric-based nano-positioners, makes it possible to compensate some of the changes that may appear in dynamic experiments. Thus, instead of working at constant applied load (or pressure), the applied load can be continuously changed in response to possible changes in surface separation, allowing to operate the system at constant separation between the surfaces, with sub-nanometric resolution. This method may improve our understanding of the dynamic response of interacting surfaces under changing conditions, like applied shear or external fields (e.g. electric or magnetic field).

To illustrate the potential of this method, in this work we studied the response of a compliant microgel-based coating under compression and shear, to investigate the coupling between normal and tangential motion and forces. Understanding of the tribology of compliant surfaces may be crucial in a number of different circumstances, from engineering (e.g. rubber seals or tires performance in wet roads) to many aspects of biotribology (e.g. ocular tribology or mammalian joints). The impact of surface compliance in surface forces can be recognized at two different levels. Under static or quasi-static conditions, surface deformation induced by surface forces increases with surface compliance, with important consequences in the way these forces are measured and analyzed. ${ }^{11,12,13,14}$ It has been recently reported how the surface deformation generated by a long range repulsive force may hinder the minimum approach between interacting surfaces, limiting the growth of tangential forces. ${ }^{15}$ Surface compliance may also have significant effects in dynamic studies; hydrodynamic forces originated by the relative motion of the surfaces may generate significant surface deformation, changing the boundary conditions of the problem. Thus, understanding the behavior of the system requires solving the coupled hydrodynamic and elasticity problems, taking into account the general force balance of the global system. This last factor requires considering the effect of the stiffness of the measuring/loading springs.

Important effects of surface compliance on dynamic surface forces studies has been documented. For the case of approaching surfaces (narrowing gaps), Leroy and coworkers showed how surface deformation engendered by the dynamic fluid pressure - a consequence of squeeze flow between moving surfaces - must be considered in the analysis of hydrodynamic interactions, even for very small deformations. ${ }^{16,17}$ Wang and coworkers investigated how elastohydrodynamic deformation 
may prevent the contact of approaching compliant surfaces, and discussed the influence of the viscoelastic properties of the walls on the system behavior. ${ }^{18}$ A study of the influence of substrate elastic deformation on hydrodynamic and pull-off forces was recently reported by Kaveh and coworkers. ${ }^{19}$

Elastohydrodynamic effects in laterally moving surfaces have been widely investigated; a historical account of elastohydrodynamic lubrication (EHL) studies was reported by Dowson. ${ }^{20}$ Different regimes of behavior in EHL can be identified, which depend on the geometry and the viscoelastic properties of both the lubricant and the confining surfaces. ${ }^{21}$ Earlier studies investigated non-conformal contact between stiff surfaces. In this condition large pressures can be readily achieved in common operational conditions, often generating an important increase in lubricant viscosity (piezoviscous effect) and hydrodynamic forces. These forces can produce significant elastic deformation of the surfaces hindering surface contact, reducing friction and wear. The daunting multiphysics problem involving the coupling between hydrodynamic and elasticity was addressed by different groups, combining theoretical and numerical methods. ${ }^{20}$ Significantly, a number of correlations involving viscoelastic properties of lubricant (viscosity) and confining surfaces (elastic modulus), and operational conditions, were proposed to successfully describe the behavior of the lubricant film thickness in EHL. Interestingly, in this regime the behavior of the system is much more sensitive to the piezoviscous properties of the lubricant than to the elastic properties of the walls.

The behavior of the system is substantially modified for the case of very compliant surfaces. In this scenario (soft EHL), surfaces can be severely deformed under load and large pressures are out of reach, so the lubricant viscosity remains constant; thus, correlations found for hard EHL do not apply. A number of theoretical studies of soft EHL have been reported. A perturbative approach to describe the limiting case of small surface deformations for different geometrical configurations was developed by Sekimoto and Leibler, ${ }^{22}$ Skotheim and Mahadevan, ${ }^{23,24}$ and others. ${ }^{25,26}$ Pandey and coworkers ${ }^{25}$ discussed the possible influence of the viscoelastic properties of the solid on soft EHL. Snoeijer and coworkers investigate the limit of large loads in soft EHL. ${ }^{27}$ The influence of $\mathrm{EH}$ effects on the motion of particles nearby deformable wall has also been discussed. ${ }^{28,29}$ Most of these studies have aimed to develop strategies to solve the combined hydrodynamic and elasticity problems, obtaining solutions for the resulting contact geometry and the magnitude of the shearinduced normal ('lift') force, due to the coupling between normal and tangential motions. On the experimental side most efforts in soft-EHL have been conducted to characterize the lubricant film thickness and the geometry of the contact in dynamic conditions. On the contrary, a small number of studies have investigated elastohydrodynamic EH lift effects. Saintyves and coworkers studied the motion of small cylinders along a soft inclined wall; ${ }^{30}$ Davies and coworkers studied moving microbeads close to compliant walls. ${ }^{31}$ In both cases, the significant effect of EH lift force on the motion was evidenced. Even more scarce are studies of EH lift on loaded contacts. Sekimoto and Leibler $^{22}$ suggested that the normal force increase observed by Klein and coworkers ${ }^{32}$ in a study of confined polymer brushes under shear can in fact be interpreted in terms of $\mathrm{EH}$ coupling. Indications of EH lift force in surfaces lubricated by compliant polyelectrolyte coatings were also reported by Bouchet et al. ${ }^{33}$

We report here the results of an experimental study of the lubrication behavior of mica surfaces coated with dense layers of thermoresponsive cationic microgels. The main novelty of the work 
was to explore the emergence of shear-induced lift forces under conditions of controlled surface separation (instead of constant applied load), providing a more accurate procedure to quantify these forces.

\section{EXPERIMENTAL SECTION}

\section{Materials}

N-Isopropylacrylamide (NIPAM), N,NMethylenebisacrylamide (MBA), aminoethyl methacrylate hydrochloride (AEMH) and 2,2'-Azobis(2-methylpropionamidine) dihydrochloride (V50) were obtained from Sigma Aldrich, and used as received.

\section{Microgel preparation}

Microgels were synthesized by batch radical polymerization following the protocol described previously $^{34}$. For the data reported in this work, the monomers NIPAM, MBA (crosslinking agent) and AEMH (aminated monomer which provides charged sites to electrostatically stabilize the microgels in water) were diluted in Milli-Q grade water (Millipore) in mass proportion 90.3/5/2.5 $(1 \mathrm{~g}$ NIPAM/50 ml). The solution was heated at $75 \mathrm{C}$ and deoxygenated under nitrogen for 1 hour. In parallel, the initiator V50 was diluted in water $(1 \mathrm{~g} / 100 \mathrm{ml})$, heated at $70 \mathrm{C}$ and deoxygenated for 10 minutes. After dissolution, the initiator solution was added to the monomer solution (2.1 gr V50/90.3 gr NIPAM), and the reaction was pursued at $75 \mathrm{C}$ under continuous nitrogen flow for 4 hours. Then, the microgel dispersion was cooled down to room temperature and purified by dialysis against deionized water during 3 days, replacing the aqueous bath three times each day. Following this procedure, microgel particles of $96 \mathrm{~nm}$ hydrodynamic radius at 25C (in $1 \mathrm{mM}$ $\mathrm{NaNO}_{3}$ ) were obtained. The mean hydrodynamic radius decreased to $81 \mathrm{~nm}$ at $35 \mathrm{C}$ and $69 \mathrm{~nm}$ at $40 \mathrm{C}$, as determined by dynamic light scattering (Malvern Zetasizer), evidencing the thermoresponsive character of the pNIPAM-based microgel. At room temperature, pNIPAM microgels are largely swollen with water; water volume fractions in hydrated microgel particles can exceed $0.9 .^{35}$

Microgel coating. The copolymerization of a fraction of cationic monomers (AEMH) in the microgels triggers electrostatically driven adsorption of the microgel on the negatively charged mica surfaces. ${ }^{36}$ Microgel adsorption was carried out during two weeks at $25 \mathrm{C}$, after an initial stage of 2 days at $35 \mathrm{C}$, in order to maximize the adsorption density microgels and the resistance of the protective coating to compression and shear. After adsorption, the microgel solution was replaced in the SFA with a $1 \mathrm{mM} \mathrm{NaNO}$ solution, to minimize electrostatic repulsion between the irreversibly adsorbed microgels. A typical AFM micrograph of the microgel-coated mica (measured ex-situ, in dry air, after the SFA experiments) is presented in the inset of the Figure 2. As can be observed in this image, a large density of physisorbed particles was present on the surfaces. We have quantified a mean value of 26 particles $/ \mu \mathrm{m}^{2}$, which corresponds to a surface coverage of ca. $75 \%$ for the swollen particles $(\mathrm{T}=25 \mathrm{C})$, signaling the high density achieved in the long microgel adsorption times implemented in this work.

\section{Surface Forces Apparatus}

We investigated the normal and friction forces between mica surfaces coated by poly ( $\mathrm{N}$ isopropylacrylamide) pNIPAM-based cationic microgel by using a Surface Forces Apparatus (SFA) modified for nanotribological studies. This technique ${ }^{37}$ is commonly used to measure the 
interaction between mica surfaces glued on silica lenses in crossed-cylinder configuration, which is equivalent to sphere-on-a-plate if the surface separation $D$ is much smaller than the radii of curvature of the surfaces, $R$. The zero separation $(D=0)$ reported in this work is determined from the thickness of the mica surfaces measured in adhesive contact in air, and corresponds to micamica contact.

Normal forces were measured from the deflection of a double cantilever springs attached to the surfaces. The static normal interaction $F_{N}$ was determined by displacing the position of the spring attached to the lower surface using a nanopositioner (P-753.2CD, Physik Instruments), and measuring the actual variation in the separation between the surfaces by MBI, as described above. The difference between both quantities indicates a variation in spring deflection, used to calculate $F_{N}$. We have implemented the method described by Heuberger and coworkers for fast $D$ calculation. ${ }^{38}$ To measure the dynamic normal (lift) force, constant speed, $V$, cyclic (back-andforth) lateral motion between the surfaces was imposed by using voltage-driven bimorph strips attached to the lower surface. ${ }^{39}$

For the measurements carried out at constant gap thickness, we have incorporated a feedback control loop based on the measured mica-mica separation at the point of closest approach (PCA)

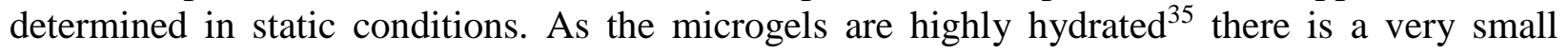
refractive index contrast with the aqueous environment. Thus, the MBI technique doesn't allow us to determine if spatial changes in coating thickness are occurring within the contact region as a consequence of a possible variation in the degree of swelling of the confined microgels. Due to variable microgel deformation in the contact (due to non-uniform pressure) the PCA in static conditions may not coincide with the closest approach between the coating layers under shear.

\section{RESULTS AND DISCUSSION \\ Normal forces. Equilibrium interactions}

Typical equilibrium (quasi-static) normal interaction force profiles between mica surfaces coated with physisorbed microgel immersed in $1 \mathrm{mM} \mathrm{NaNO} 3$ bath are presented in Figure 2. As described before, the reported distance is referred to the mica-mica contact measured in dry air; the mica surfaces were kept inside the SFA in an aqueous environment at all times, during and after microgel adsorption. The force profiles were measured slowly approaching the surfaces towards each other, in steps of the position $T$ of $5 \mathrm{~nm}$. The surfaces were kept at rest 20 seconds between two experimental points, to assure the data were obtained at equilibrium. A long-range, repulsive interaction exponentially decreasing with the distance between the surfaces was observed, due to the steric interaction between the adsorbed particles. As expected, the range of the interaction is strongly dependent on microgel swelling, determined by the temperature. Thus, while at $\mathrm{T}=25 \mathrm{C}$ the onset of repulsive interaction was observed with the mica surfaces about $400 \mathrm{~nm}$ apart, it was reduced to about $250 \mathrm{~nm}$ at $\mathrm{T}=35 \mathrm{C}$. The distance at the onset of repulsion, $D_{\text {onset }}$, can be related to the temperature-dependent effective thickness of the microgel coating by $H_{s}=D_{\text {onset }} / 2$. Nevertheless, it is important to keep in mind that there is a certain degree of polydispersity in the microgels, which are porous, hydrated objects adsorbed as independent entities. In this sense, there is a large degree of uncertainty on the definition of $H_{s}$.

The system under study is a multilayer system, with layers (glue, mica and microgel) of different elastic properties. By increasing the applied pressure (increasing the normal load $L$ ), the different 
layers will deform; the compliant microgels will be compressed and shrunk between the mica surfaces; as the contact is non-conformal, this deformation will be a function of the position in the contact region. Likewise, the glue under the mica will deform under pressure, which is also spatially variant, from a maximum value at the point of closest approach to ambient pressure outside of the contact region. The long-range repulsive force due to the compression of the microgel coating must be taken into account to describe the contact mechanics of the system, and to correctly interpret the surface force measurements. ${ }^{11-14}$ It has been recently described how this long range repulsive force, together with the mechanics of the contact, may have important consequences on the behavior of this system under compression and shear. ${ }^{15}$

\section{Normal forces: draining}

The normal interaction force profiles presented in Figure 2 were measured in quasi-static conditions, allowing the surfaces and microgel coating to reach equilibrium steady-state for each experimental point. The scenario in dynamic (out-of-equilibrium) conditions can be substantially different. In particular, some transient opposition to the surface motion appears due to the viscous resistance to fluid flow perpendicular to the applied pressure during draining, as water must flow out of the compressed microgels (microgel are porous and hydrated in the absence of stress). This flow, which involves transport of water molecules over tens of nanometers, depends on the size of the system. A poroelastic time can be defined, characterizing the migration time of water molecules across the contact region. This poroelastic time depends on the contact size and can be as long as few hours for sizes of the order of few centimeters, as extensively discussed by several groups. ${ }^{40,41}$

To evaluate the typical poroelastic time of our system, we have studied the response of the system to large $T$ displacements (ca. $1 \mu \mathrm{m}$ ) at different velocities. Typical results are presented in Figure 3. At slow speeds and large separations the surfaces approached at the same rate as the imposed normal velocity; closer in, the approach slows down due to the emergence of the steric interaction force. Nevertheless, the deviation from linearity maps the contribution of the static interaction force, indicating that the repulsive hydrodynamic interaction is not significant. For the fastest change of $T$ investigated $(1.3 \mu \mathrm{m} / \mathrm{s}), D$ is quickly reduced, reaching separations below $130 \mathrm{~nm}$ (the thinnest film investigated in dynamic conditions below) in less than $1 \mathrm{sec}$, and the steady-state separation in few seconds. These results evidence a small viscous resistance to the imposed displacement, even in the cases of strong microgel compression, suggesting that the characteristic poroelastic time is shorter than $1 \mathrm{~s}$ (the temporal resolution of our experimental setup) for $D>130$ $\mathrm{nm}$. This short time is due in part to the small size of the contact investigated (few tens of microns) but mainly to the rapid response of the microgels, due to its submicrometric size. The fast swellingshrinking time of pNIPAM-based microgels have been discussed before. ${ }^{42}$

\section{Tangential motion}

The lateral relative motion of the surfaces breaks the radial symmetry of the problem and pushes the system to out-of-equilibrium conditions. The possibility of continuous, real-time monitoring of the contact geometry allows to achieve a better understanding of the dynamical system. We investigated the behavior under shear of the microgel-coated mica in two different settings. First, an initial external normal load $L$ was imposed using the loading spring, and then the position $T$ remained unchanged. In a second set of experiments, the response of the system to the applied shear was investigated under constant mica-mica separation by implementing the feedback control loop described above, changing the position of $T$ to regulate the applied normal force. The results 
reported in this communication are related to the coupling between normal and lateral motion (shear-induced lift force), seemingly due to elastohydrodynamic effects.

From our experimental setup, we can not explore the detailed behavior of the microgel particles under shear. In particular, we are not able to determine if sliding or rolling motion of the particles is occurring inside the contact region. However, no evidence of shear-induced wear or irreversible changes was detected in any of the contacts investigated. Thus, no noticeable changes were observed in the normal interaction force profiles measured before and after shear.

\section{Shear-induced lift force: constant position of distal spring end, $T$}

Typical results for the shear-induced variation of the separation of the point of closest approach between the surfaces at different values of $V$ are presented in Figure 4. In general, a strong $V$ dependent variation of the PCA was observed at all surface separations below ca. $300 \mathrm{~nm}$, further appart than the supposed contact between the microgel layers in the static approach. This dilation, which is necessarily accompanied by the compression of the loading spring, was more significant at higher temperatures. Thus, as the hydrodynamic size (and hydration degree) of the microgels decreased $(\mathrm{T}=35 \mathrm{C})$, the shear-induced lift force, $F_{\text {liff, }}$ increased. This important point will be addressed below.

The position of $T$ remains unchanged during the surface displacement; thus, the variation in separation $(\Delta D)$ implies further deflection of the normal spring, i.e., a change in $F_{\text {spring }}$ (which in static conditions is identified as the normal load, $L$ ). In static conditions, a non-zero elastic $F_{\text {spring }}$ is balanced by steric contact forces. On the contrary, when $D$ increases upon surface motion, fluid is entrained between the moving surfaces; the steady-state $F_{\text {spring }}$ is balanced by elastohydrodynamic forces if a fluid film is present between the surfaces. This is the case at large separations, when the microgels are out of contact; but even at conditions of static contact between the microgels (non-zero static load $L$ ), the fact that under dynamic conditions $F_{\text {spring }}$ increases significantly, strongly suggests that a fluid film is being developed between the surfaces due to hydrodynamic effects. Under this scenario, the system transits from boundary to hydrodynamic lubrication. In the conditions of low Reynolds number investigated in this work, Stokes equation applies. It has often been discussed that as a consequence of the reversibility of Stokes flow, no hydrodynamic normal force can be generated in the symmetric geometry investigated in this work (sphere-on-a-plate contact). ${ }^{24,22,43}$ This scenario changes when elastic deformation of the walls breaks the symmetry of the contact: growing deformation of the boundaries engenders a greater asymmetry of the pressure distribution in the contact region, leading to a non-null lift force between the rubbing surfaces.

We can use the value of $\Delta D$ to obtain an estimation of $F_{\text {lift, }}$ which is a continuously increasing function of $V$. However, several factors must be taken into account. First, the microgel coating changes during an experiment due the changing degree of confinement. This variation will modify the extent of swelling and in consequence the elastic properties of the coating. Second, the varying mica-mica separation will make it difficult to precisely define the applied shear rate. Third, as the normal spring deflection is changing during the experiment, both the applied load and $D$ vary. In addition, as $F_{\text {lift }}$ is a function of the experimental conditions ( $V$ and $\left.D\right), \Delta D$ will be a function of the stiffness of the experimental setup (i.e., the spring constant of the apparatus). All these factors will complicate the modelling and theoretical description of the system under study, and the comparison between results of different laboratories. 


\section{Shear-induced lift force: constant separation $D$}

For the reasons mentioned above, we have implemented a feed-back system to allow a precise control of the distance $D$ between the mica surfaces, by varying the position $T$. Using MBI as described above, the distance between the mica surfaces (at the PCA) can be controlled with an accuracy of $0.1 \mathrm{~nm}$. As $D$ remains unchanged, $\Delta T$ can be directly used to calculate the velocitydependent $F_{l i f t}$ in controlled conditions of $D$ and nominal shear rate $(V / D)$.

Typical experimental traces are presented in Figure 5. The applied velocity was abruptly changed at regular intervals, as indicated. As described before, each time $V$ was augmented the separation between the surfaces raised. However, by applying the adequate correction to the position $T$, the separation between the mica surfaces at steady-state was kept at a fixed value. After $D$ reaches the set-point, $\Delta T$ can be translated in a changing spring deflection, allowing to readily quantify the velocity-dependent lift force under better controlled conditions than in the preceding case. Due to the limited range of lateral displacement of our setup, a reciprocating motion was applied during the experiments. However, by testing different combinations of shear amplitude and frequency, it was established that the driving speed $V$ is the main variable determining the shear-induced $F_{\text {lift. }}$.

As it is necessary to increase $F_{\text {spring }}$ to maintain a constant gap, the average pressure inside the contact increases under shear, which compresses further the microgel coating (compared with the initial static condition). The compression of the microgel coating at constant $D$ strongly suggests the emergence of a lubricant fluid film between the surfaces at sufficiently large $V$. Thus, while in static conditions $F_{\text {spring }}$ is balanced by surface forces, under motion the enhanced $F_{\text {spring }}$ is balanced by repulsive forces of hydrodynamic origin: if full fluid film conditions are assumed, the entire load is carried by it and $F_{\text {spring }}$ is equivalent to $F_{\text {lift }}$, an integration of the liquid film pressure in the contact area.

It is apparent that this method allows a clearer description of the system under study. However, one point of warning is in order. The lift force measured is a macroscopic value that integrates the contribution of the whole contact area. However, as mentioned before, the shear-induced pressure is a function of the position in the contact. Thus, it produces a deformation of the microgel coating, which is a function of the position in the contact region. Even though we are imposing the closest separation between the mica surfaces, the distance between the microgel coatings is not constant, due to the position-dependent microgel deformation. In other words, we are able to control the separation between the mica surfaces; not the separation between the deformable microgel coating layers.

Typical results for the shear-induced variation in $F_{\text {spring }}$ (calculated as $\Delta T . K_{N}$ ) as function of the surface velocity $V$ for different inter-surface distances are presented in Figure 6. As can be observed in the figure, the measured lift force increases significantly with driving speed (nearly proportional to the square of $V$ ), and decreases with the gap between the surfaces. From our definition of coating thickness based in the normal interaction force profile, $H_{S}$, the microgel coatings are in static contact for separations smaller than $250 \mathrm{~nm}$. However, to maintain a constant gap while shearing, $F_{\text {spring }}$ (and the mean gap pressure) must be augmented, which reduces the effective microgel thickness, as illustrated by the schema in the inset of Fig.6. We can not measure directly the thickness of the microgel coating by MBI because it is highly hydrated, with little 
contrast of refractive index with the aqueous environment. A crude estimate of the dynamic microgel film thickness $H_{l k}$ can be obtained from the static force profile (Fig. 2), making $H_{l k}$ equal to half of the separation expected for an applied normal force corresponding the dynamic $F_{\text {spring. }}$. Under this assumption the thickness of the fluid film developed under shear, $h_{0}$, can be approximated as $h_{0=} D-2 . H_{l k}$.

The evolution of the measured forces with the mica-mica distance is presented in Figure 7. In Fig. 7a we compare the static normal force with the dynamic variation of $F_{\text {spring }}$ at the fastest $V$ investigated $(V=9.6 \mu \mathrm{m} / \mathrm{s})$. As can be observed in the Figure, the shear-induced variation in $F_{\text {spring }}$ ( $\Delta F_{\text {spring }}$ ) is larger than the static interaction force for large gaps, but this difference is reduced at shorter separations. However, $\Delta F_{\text {spring }}$ can not be directly equated to the dynamic lift force: as mentioned before, if a fluid film is completely developed between the surfaces, the repulsive steric force will not be present. In the absence of this steric force, $F_{\text {spring }}$ will be entirely balanced by the hydrodynamic lift force $\left(F_{\text {lift }}=F_{\text {spring }}\right)$. The lift force determined by this method as a function of the liquid gap $h_{0}$ determined as described in the previous paragraph is presented in Figure $7 \mathrm{~b}$. As can be observed in the figure, the measured lift force decreases quickly with the thickness of the fluid gap, as expected for EH effects. The validity of the EH origin of the observed lift force could be further evaluated by comparing the evolution of the measured force on the different operational variables with theoretical predictions. Describing the dynamic system of a lubricated, compliant, point contact requires solving the coupled non-linear equations of hydrodynamic and elasticity, a very challenging problem due to the non-local effects introduced by the elastic deformation. We are not aware of any exact theoretical description for the full analytical description of this problem. Sekimoto and Leibler ${ }^{22}$ and Skotheim and Mahadevan ${ }^{23,24}$ used a perturbation approach to develop analytical expressions for the EH lift force in the scenario of small deformations. For a sphereplane lubricated contact of a thin poroelastic layer on a rigid substrate, they proposed:

$$
F_{\text {lift }}=\frac{\mu^{2} V^{2} H_{l} R^{2}}{(2 G+\lambda) h_{0}^{3}}
$$

where $\mu$ is the fluid viscosity, and $G$ and $\lambda$ are the shear modulus and the Lamé's first parameter of the thin layer. As this expression is valid in the limit of small deformations, it does not apply strictly to the system investigated here, in particular in the regime of large compressions. Nevertheless, we can compare it with our experimental results. As can be observed in Figure 6, the measured $F_{\text {lift }}$ data grows following a square dependence on $V$, in agreement with the previous expression. On the contrary, it clearly decays more slowly than the third power of the estimated thickness of the lubricant film (the prediction of eq. 1), as can be observed in Figure 7. One identifiable reason for this discrepancy comes from the fact that the elastic shear modulus of the microgel and its thickness are both strongly affected on the degree of swelling. Thus, the factor $(2 G+\lambda)$ in the denominator and $H_{l}$ in the numerator of (1) are not independent of $D$. As a first approximation, one can consider the microgel coating thickness $H_{l}$ as the minimum between $H_{s}$ and half of the mica-mica separation before shear. It can also be assumed that the shear modulus is proportional to the density of network strands per unit volume, ${ }^{44}$ which is inversely proportional to $H_{l}$. As can be observed in Figure 8, by scaling the measured $F_{\text {lift }}$ data by $H_{s} / H_{l}{ }^{2}$, a reasonably agreement with an inverse cubic power law of $h_{0}$ is obtained, as predicted by equation 1. 
A more rigorous test to the description provided by eq. 1 comes from the direct calculation of microgel shear modulus from the experimental data. We obtains values of $(2 G+\lambda)$ ca. $1 \mathrm{~Pa}$, which appears unreasonably low: direct measurements of the modulus of similar microgel systems are typically of the order of few $\mathrm{kPa} .{ }^{45}$ Several reasons could be at the origin of this discrepancy. First, the actual viscosity of the lubricant film is unknown; we have considered this film to be a layer of water, but it is probably more adequate to picture it as a solution of pNIPAM pendant chains. Indeed, it has been shown that the internal microstructure of common microgels, and in particular their degree of cross-linking, is often non-homogeneous. ${ }^{46,47}$ By using neutron scattering experiments, Stieger and coworkers showed that the crosslinking density of pNIPAM microgels decreases radially in the particle; thus, it is probably a more accurate image to consider the microgel layer as an elastic core decorated with a layer of dangling pNIPAM chains. Other reasons for calculating unrealistically low values of $G$ could be the overestimation of the values of $h_{0}$ (from values of $D$ and $F_{\text {lift }}$ measured under dynamic conditions) or the large microgel deformation used in this work: large strains were applied in most of the test reported, which is beyond the range of validity of eq. 1, developed for small perturbations. Nevertheless, we obtained small $G$ values even for the cases of none or small compression of the microgel layer $\left(D / 2 \geq H_{s}\right)$.

Another point of discrepancy between our experimental results and the model described by eq. 1 is the behavior observed at different temperatures. We found a significantly enhanced $F_{\text {lift }}$ at higher temperatures (Fig. 4), when the swelling degree of the microgels is clearly reduced (cf. Fig 2). However, if eq. 1 holds, the opposite is expected, as the shear modulus of the microgel significantly decreases in the more hydrated state. ${ }^{45}$ This apparent inconsistency may be partially due to the permeability of the microgel coating. Salez and Mahadevan ${ }^{28}$ argued that for short poroelastic times (compared with the lubrication time scale) this element can be taken into account by including a (1- $\phi$ ) factor in equation 1 (where $\phi$ is the fluid volume fraction in the porous layer), implying an effective stiffening due to the presence of the fluid. This stiffening would be more significant at lower temperatures, when the degree of swelling of the microgel coating increases. Another important element missing in the description of Skotheim and Mahadevan is the influence of wall viscoelasticity. ${ }^{23,24}$ As described by Pandey and coworkers ${ }^{25}$, the rheological properties of the walls can modify the behavior of the system; in particular, smaller $F_{\text {lift }}$ values should be expected for viscoelastic boundaries than for purely elastic system due to the dissipative contribution of the wall loss modulus.

Another remaining question of this study is the actual existence of a fluid lubricant film between the surfaces under compression and shear. There is no doubt that it will be present at large separations $\left(D>H_{s}\right)$, but it may not be the case under an applied normal load. Working on macroscopic hydrogels, Chateauminois and coworkers ${ }^{41}$ found that the system behavior was dominated by the long poroelastic times, and did not observe the formation of a lubricant film, reducing the influence of $\mathrm{EH}$ effects. On the contrary, in the present study the poroelastic time was short related to the typical excitation time $\left(\tau \approx A^{1 / 2} / V\right)$ due to the small size of the contact and the microgels; we observed the emergence of a lift force due to the coupling between normal and tangential motion: when the mechanical system was left to evolve freely, we observed a significant increase of the gap between the surfaces. Thus, our results strongly suggests that under shear the load bearing ability in the system progressively shifts from steric forces (surfaces in contact; boundary lubrication) to hydrodynamic forces of EH origin with increasing driving velocities, with a lubricant film separating the rubbing surfaces. This transit could be responsible for the bell- 
shaped response of friction $v s . V$ (with a maximum value) often observed in lubrication studies of macromolecular coatings. ${ }^{33}$

\section{CONCLUSIONS}

We have shown that shearing of surfaces coated by compliant microgel layers can generate a lift force of elastohydrodynamic origin, triggering the entrainment of a fully developed fluid film at large enough speeds, minimizing the contact between the opposite surfaces. After fluid film formation the system transits from boundary to hydrodynamic modes of lubrication, which is likely to trigger significant friction and wear reduction.

By using a feedback loop to fix the surface separation, we have been able to accurately determine the lift force, and to investigate its dependence on driving speed and surface separation. The scaling of the lift with these variables can be reasonably described by perturbative models developed to describe small dynamic deformation of the compliant coating. 

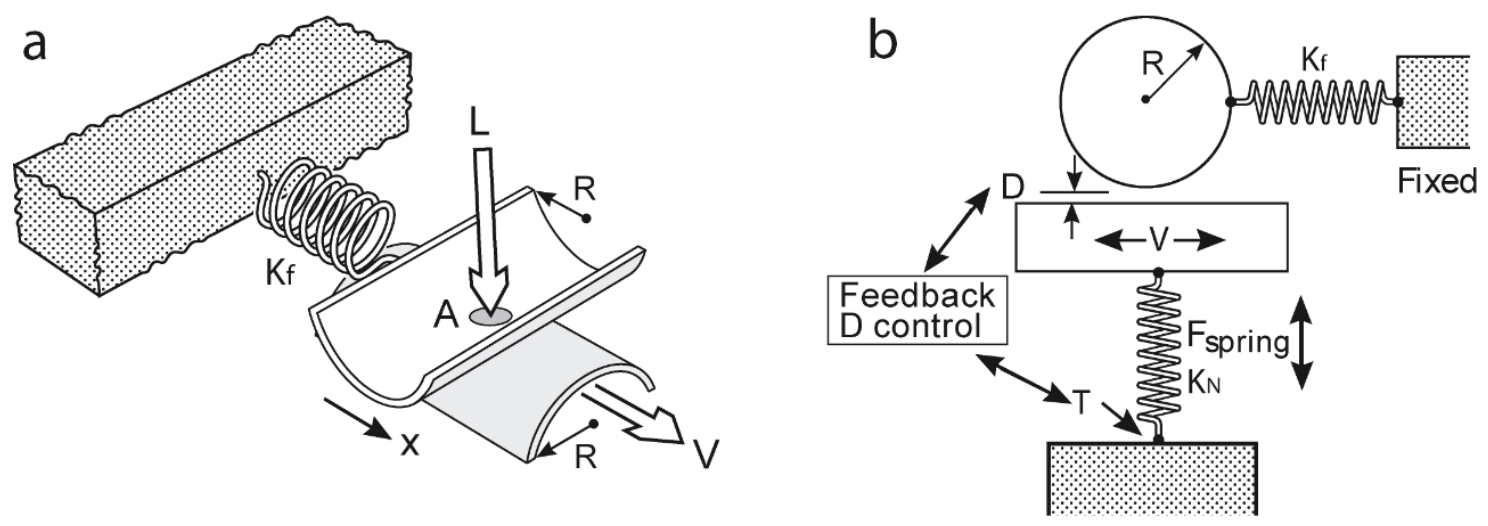

Figure 1. Schematic of the mechanism of the Surface Forces Apparatus. Two mica surfaces are glued on cylindrical lenses of radius $R$ with their axis crossed. The distance $D$ between the surfaces is measured by MBI. A normal force $\left(F_{\text {spring }}\right)$ is applied between the surfaces using the normal spring $\left(K_{N}\right)$, by displacing the distal position $T$. This force determines the position $D$. The point of contact grows to a circular contact area $A$ under load. The reciprocating tangential motion between the surfaces at a speed $V$ is imposed by using piezoelectric actuators. The tangential force between the surfaces is determined from the deflection of the tangential spring $K_{f}$

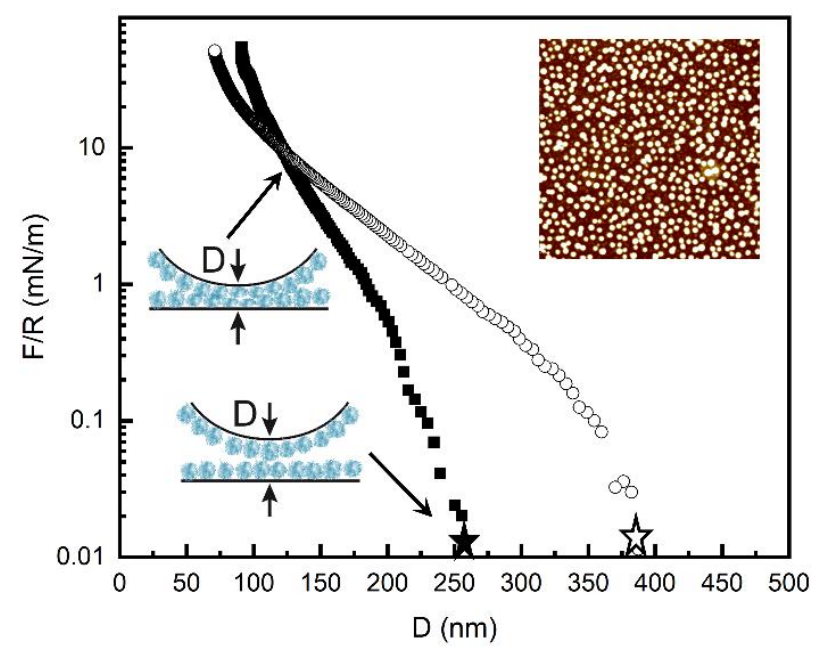

Figure 2. Normal interaction force measured during quasi-static approach (normalized by the nondeformed radius of curvature of the surfaces) vs. surface separation $D$ between mica surfaces coated with a self-assembled layer of cationic pNIPAM microgels immersed in a solution of $1 \mathrm{mM}$ $\mathrm{NaNO}_{3}$. Black squares: $\mathrm{T}=35 \mathrm{C}$. Open circles: $\mathrm{T}=25 \mathrm{C}$. The stars signal the onset of repulsive force, which can be identified as twice the thickness of the unperturbed microgel coating $\left(H_{s}\right)$. Inset: $5 \mu \mathrm{m} \times 5 \mu \mathrm{m}$ AFM tapping mode height micrograph measured in air (ex-situ) of a dried microgel-coated mica surface, after the SFA experiments. 


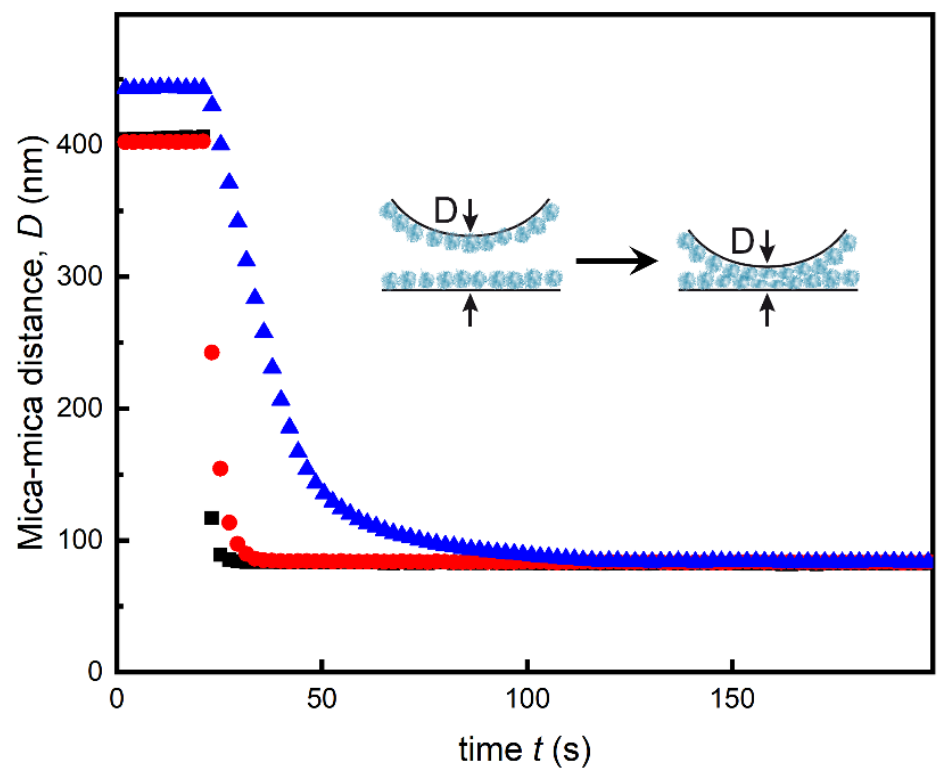

Figure 3. Liquid film draining: variation of the mica-mica distance at the PCA. An inward displacement of $T$ of $1.3 \mu \mathrm{m}$ was imposed at $\mathrm{t}=20 \mathrm{~s}$ at different speeds. Black squares: $1.3 \mu \mathrm{m} / \mathrm{s}$. Red circles: $0.13 \mu \mathrm{m} / \mathrm{s}$. Blue triangles: $0.013 \mu \mathrm{m} / \mathrm{s}$. $\mathrm{T}=25 \mathrm{C}$.

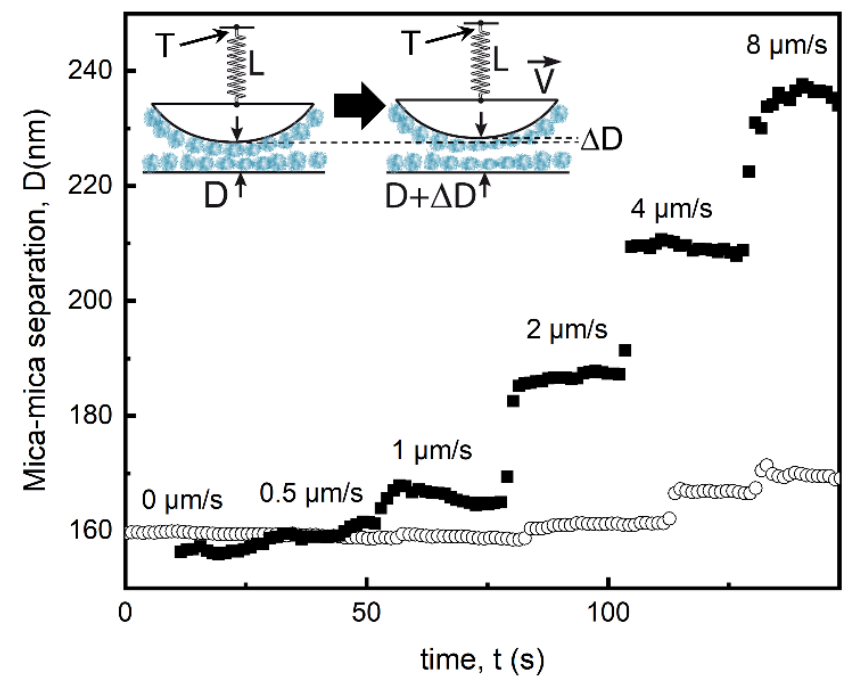

Figure 4. Shear-induced variation of the mica-mica distance at the PCA under static conditions. Black squares: $\mathrm{T}=35 \mathrm{C}$. Open circles: $\mathrm{T}=25 \mathrm{C}$. The driving velocity $V$ was modified at periodic intervals, as specified. The distal point of the loading spring $T$ remains in a fixed position. 


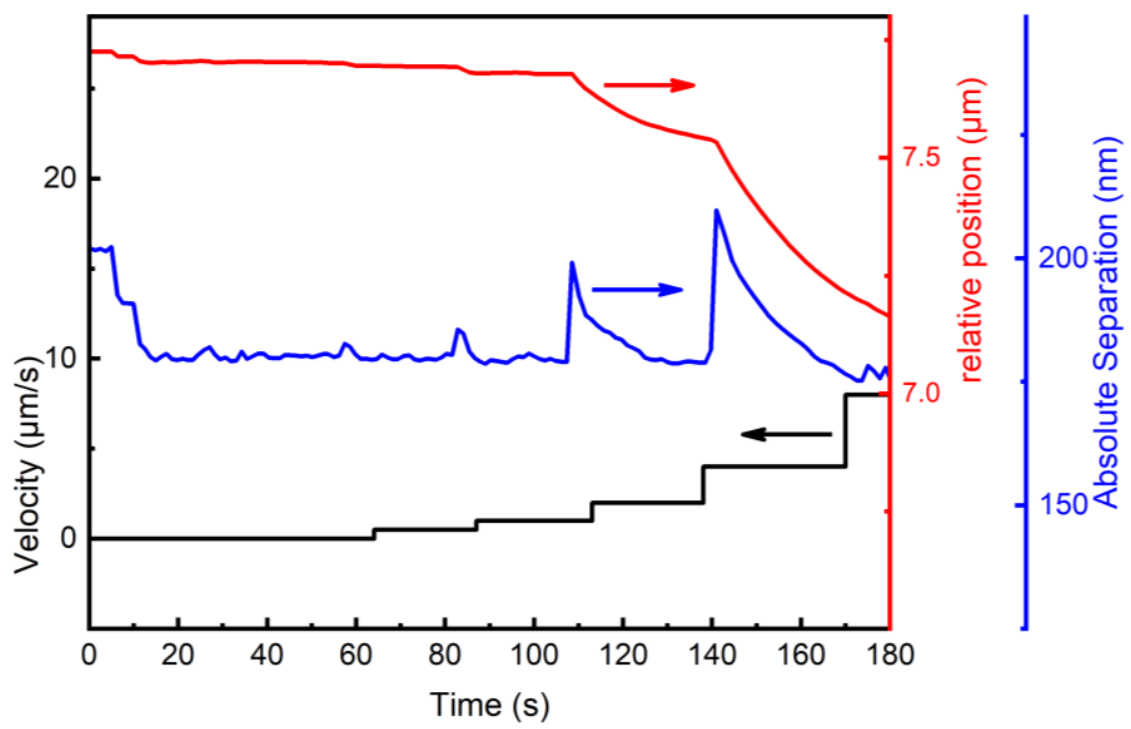

Figure 5. Feedback loop control. The distal point of the loading spring is displaced to maintain the target value of the mica-mica distance at the PCA. Black line: applied driving speed $V$. Blue line: measured absolute mica-mica distance, $D$. Red line: relative position of the distal point of the loading spring, $T$. $\mathrm{T}=35 \mathrm{C}$

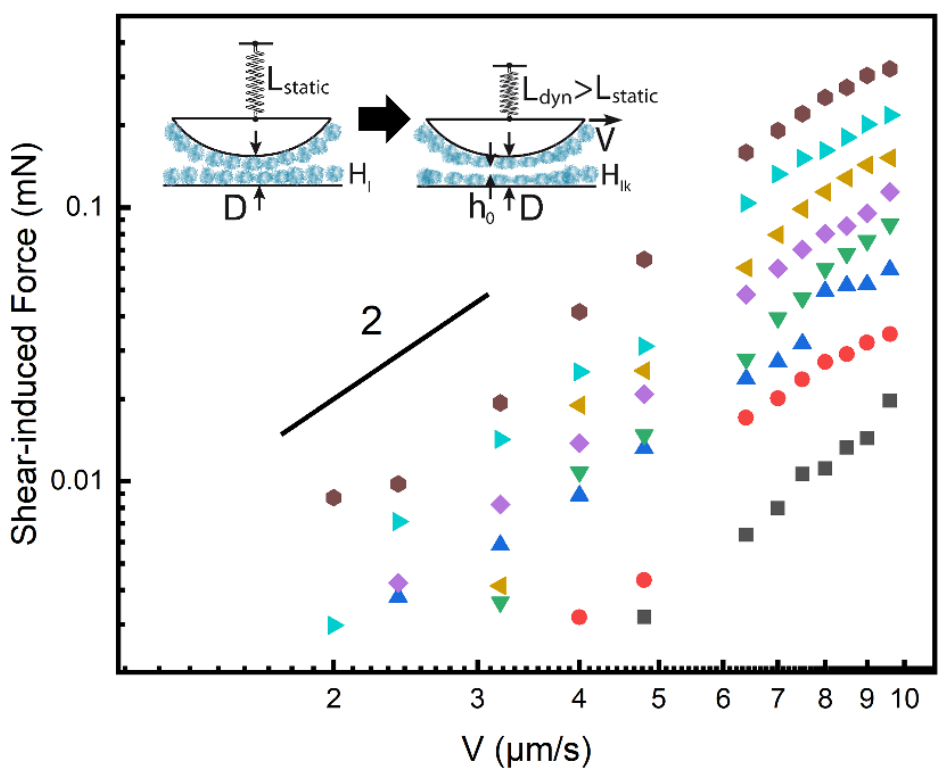

Figure 6. Shear-induced lift force vs. driving speed at different imposed mica-mica distance $D$. Black squares, $D=300 \mathrm{~nm}$; red circles, $D=250 \mathrm{~nm}$, blue triangles $D=240 \mathrm{~nm}$, green inverted triangles $D=210$, purple rhombi $D=190 \mathrm{~nm}$, ochre triangles $D=180 \mathrm{~nm}$, cyan triangles $D=160$ $\mathrm{nm}$, brown hexagons $D=130 \mathrm{~nm}$. T=35 C 

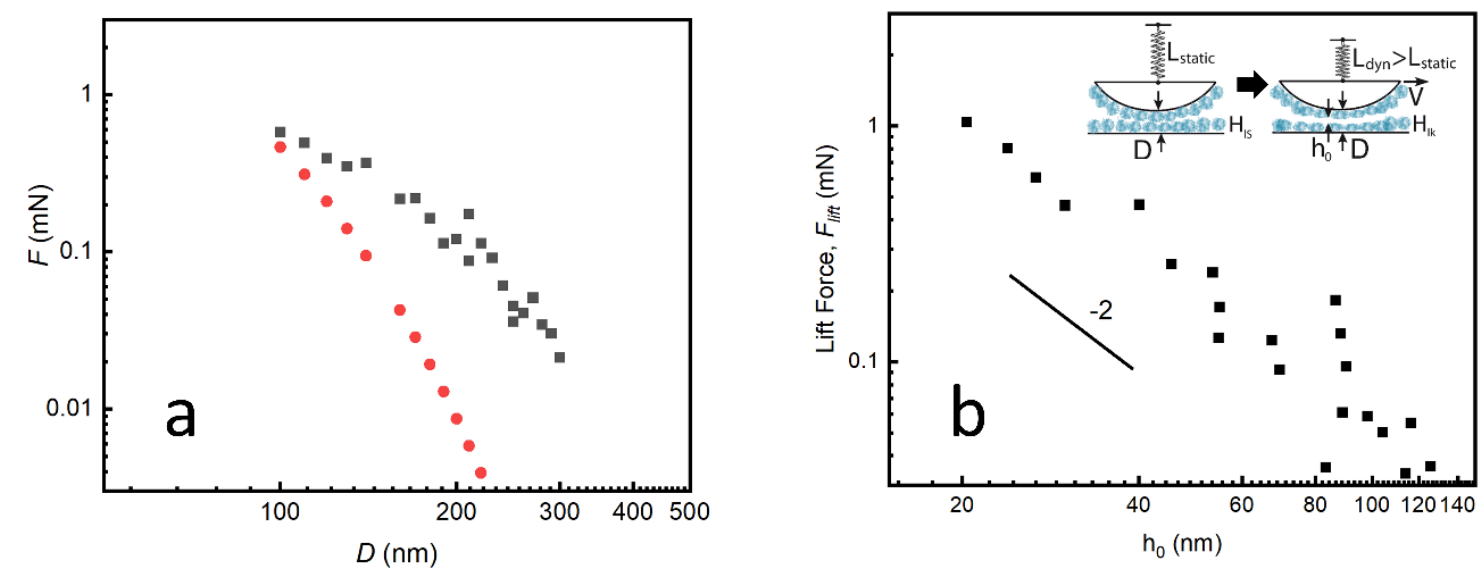

Figure 7. a) Static normal interaction force (red circles) and dynamic shear-induced variation in normal force, $\Delta F_{\text {spring }}$ (black squares) vs. $D ; V=9.6 \mu \mathrm{m} / \mathrm{s} ; \mathrm{T}=35 \mathrm{C}$. b) Lift force vs. thickness of the shear-induced fluid film. $V=9.6 \mu \mathrm{m} / \mathrm{s} ; \mathrm{T}=35 \mathrm{C}$

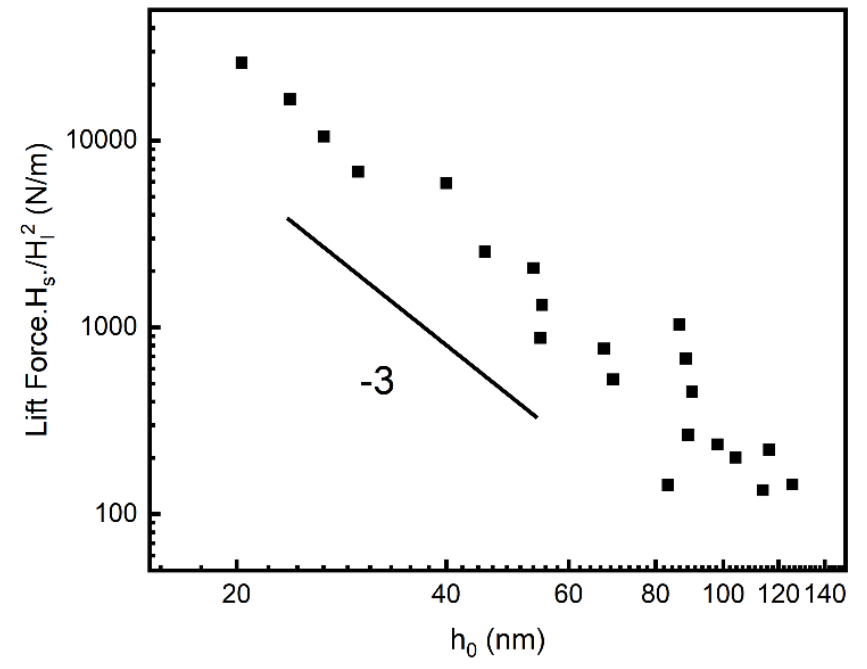

Figure 8. a) Product of the Lift force by the compression-induced relative variation in shear modulus $\left(H_{s} / H_{l}\right)$, divided by the thickness of the microgel film $\left(H_{l}\right)$ vs. thickness of the shearinduced fluid film. $V=9.6 \mu \mathrm{m} / \mathrm{s} ; \mathrm{T}=35 \mathrm{C}$ 


\section{ACKNOWLEDGMENT}

The authors are indebted to Dr. T. Salez for stimulating discussions and suggestions on the manuscript.

\section{REFERENCES}

(1) Tomlinson, G. A. LXVII. Molecular Cohesion. London, Edinburgh, Dublin Philos. Mag. J. Sci. 1928, 6 (37), 695-712.

(2) Overbeek, J. T. G.; Sparnaay, M. J. Classical Coagulation. London-van Der Waals Attraction between Macroscopic Objects. Discuss. Faraday Soc. 1954, 18 (1), 12.

(3) Rabinovich, Y. I.; Derjaguin, B. V.; Churaev, N. V. Direct Measurements of Long-Range Surface Forces in Gas and Liquid Media. Adv. Colloid Interface Sci. 1982, 16 (1), 63-78.

(4) Bailey, A. I.; Cortney-Pratt, J. S. The Area of Real Contact and the Shear Strength of Monomolecular Layers of a Boundary Lubricant. Proc. R. Soc. London. Ser. A. Math. Phys. Sci. 1955, 227 (1171), 500-515.

(5) Israelachvili, J. N.; Tabor, D. The Measurement of Van Der Waals Dispersion Forces in the Range 1.5 to 130 Nm. Proc. R. Soc. A Math. Phys. Eng. Sci. 1972, 331 (1584), 19-38.

(6) Israelachvili, J. N.; Adams, G. E. Measurement of Forces between Two Mica Surfaces in Aqueous Electrolyte Solutions in the Range 0-100 Nm. J. Chem. Soc. Faraday Trans. 1 1978, 74, 975-1001.

(7) Israelachvili, J. N.; Physics, S. Thin Film Studies Using Multiple-Beam Interferometry. J. Colloid Interface Sci. 1973, 44 (2), 259-272.

(8) Tonck, A.; Georges, J. M.; Loubet, J. L. Measurements of Intermolecular Forces and the Rheology of Dodecane between Alumina Surfaces. J. Colloid Interface Sci. 1988, 126 (1), $150-163$.

(9) Stewart, A. M.; Parker, J. L. Force Feedback Surface Force Apparatus: Principles of Operation. Rev. Sci. Instrum. 1992, 63 (12), 5626.

(10) Parker, J.; Stewart, A. Force Feedback Measurements of Surface Forces. Prog. Colloid Polym. Sci. 1992, 88, 162-168.

(11) Hughes, B. D.; White, L. R. Implications of Elastic Deformation on the Direct Measurement of Surface Forces. J. Chem. Soc. Faraday Trans. 1 Phys. Chem. Condens. Phases 1980, 76, 963.

(12) Parker, J. L.; Attard, P. Deformation of Surfaces Due to Surface Forces. J. Phys. Chem. 1992, 96 (25), 10398-10405.

(13) Attard, P.; Parker, J. L. Deformation and Adhesion of Elastic Bodies in Contact. Phys. Rev. 
A 1992, 46 (12), 7959-7971.

(14) Barthel, E. Surface Deformation, Spring Stiffness and the Measurement of Solvation Forces. Thin Solid Films 1998, 330 (1), 27-33.

(15) Vialar, P. V.; Merzeau, P.; Barthel, E.; Giasson, S.; Drummond, C. Interaction between Compliant Surfaces: How Soft Surfaces Can Reduce Friction. Submitted 2019.

(16) Leroy, S.; Charlaix, E. Hydrodynamic Interactions for the Measurement of Thin Film Elastic Properties. J. Fluid Mech. 2011, 674, 389-407.

(17) Leroy, S.; Steinberger, A.; Cottin-Bizonne, C.; Restagno, F.; Léger, L.; Charlaix, I. Hydrodynamic Interaction between a Spherical Particle and an Elastic Surface: A Gentle Probe for Soft Thin Films. Phys. Rev. Lett. 2012, 108 (26), 1-5.

(18) Wang, Y.; Dhong, C.; Frechette, J. Out-of-Contact Elastohydrodynamic Deformation Due to Lubrication Forces. Phys. Rev. Lett. 2015, 115 (24), 1-5.

(19) Kaveh, F.; Ally, J.; Kappl, M.; Butt, H. J. Hydrodynamic Force between a Sphere and a Soft, Elastic Surface. Langmuir 2014, 30 (39), 11619-11624.

(20) Dowson, D. Elastohydrodynamic and Micro-Elastohydrodynamic Lubrication. Wear 1995, 190 (2), 125-138.

(21) Johnson, K. L. Regimes of Elastohydrodynamic Lubrication. J. Mech. Eng. Sci. 1970, 12 (1), 9-16.

(22) Sekimoto, K.; Leibler, L. A Mechanism for Shear Thickening of Polymer-Bearing Surfaces: Elasto-Hydrodynamic Coupling. Europhys. Lett. 1993, 23 (2), 113-117.

(23) Skotheim, J.; Mahadevan, L. Soft Lubrication. Phys. Rev. Lett. 2004, 92 (24), 245509.

(24) Skotheim, J. M.; Mahadevan, L. Soft Lubrication: The Elastohydrodynamics of Nonconforming and Conforming Contacts. Phys. Fluids 2005, 17 (9), 092101.

(25) Pandey, A.; Karpitschka, S.; Venner, K.; Snoeijer, J. Lubrication of Soft Viscoelastic Solids. 2015, 433-447.

(26) Urzay, J.; Llewellyn Smith, S. G.; Glover, B. J. The Elastohydrodynamic Force on a Sphere near a Soft Wall. Phys. Fluids 2007, 19 (10).

(27) Snoeijer, J. H.; Eggers, J.; Venner, C. H. Similarity Theory of Lubricated Hertzian Contacts. Phys. Fluids 2013, 25 (10), 23-29.

(28) Salez, T.; Mahadevan, L. Elastohydrodynamics of a Sliding, Spinning and Sedimenting Cylinder near a Soft Wall. J. Fluid Mech. 2015, 779, 181-196.

(29) Saintyves, B.; Jules, T.; Salez, T.; Mahadevan, L. Self-Sustained Lift and Low Friction via Soft Lubrication. Proc. Natl. Acad. Sci. 2016, 113 (21), 5847-5849. 
(30) Saintyves, B.; Jules, T.; Salez, T.; Mahadevan, L. Self-Sustained Lift and Low Friction via Soft Lubrication. Proc. Natl. Acad. Sci. 2016, 113 (21), 5847-5849.

(31) Davies, H. S.; Débarre, D.; El Amri, N.; Verdier, C.; Richter, R. P.; Bureau, L. Elastohydrodynamic Lift at a Soft Wall. Phys. Rev. Lett. 2018, 120 (19), 2-7.

(32) Klein, J.; Perahia, D.; Warburg, S. Forces between Polymer-Bearing Surfaces Undergoing Shear. Nature 1991, 352 (6331), 143-145.

(33) Bouchet, A. S.; Cazeneuve, C.; Baghdadli, N.; Luengo, G. S.; Drummond, C. Experimental Study and Modeling of Boundary Lubricant Polyelectrolyte Films. Macromolecules 2015, $48(7), 2244-2253$.

(34) López-León, T.; Ortega-Vinuesa, J. L.; Bastos-González, D.; Elaïssari, A. Cationic and Anionic Poly( N -Isopropylacrylamide) Based Submicron Gel Particles: Electrokinetic Properties and Colloidal Stability. J. Phys. Chem. B 2006, 110 (10), 4629-4636.

(35) Yang, M.; Liu, C.; Zhao, K. Concentration Dependent Phase Behavior and Collapse Dynamics of PNIPAM Microgel by Dielectric Relaxation. Phys. Chem. Chem. Phys. 2017, 19 (23), 15433-15443.

(36) Pérez-Fuentes, L.; Drummond, C.; Faraudo, J.; Bastos-González, D. Anions Make the Difference: Insights from the Interaction of Big Cations and Anions with Poly(NIsopropylacrylamide) Chains and Microgels. Soft Matter 2015, 5077-5086.

(37) Israelachvili, J.; Min, Y.; Akbulut, M.; Alig, A.; Carver, G.; Greene, W.; Kristiansen, K.; Meyer, E.; Pesika, N.; Rosenberg, K.; et al. Recent Advances in the Surface Forces Apparatus (SFA) Technique. Reports Prog. Phys. 2010, 73 (3), 036601.

(38) Heuberger, M. The Extended Surface Forces Apparatus. Part I. Fast Spectral Correlation Interferometry. Rev. Sci. Instrum. 2001, 72 (3), 1700-1707.

(39) Luengo, G.; Schmitt, F.-J.; Hill, R.; Israelachvili, J. Thin Film Rheology and Tribology of Confined Polymer Melts: Contrasts with Bulk Properties. Macromolecules 1997, 30 (8), 2482-2494.

(40) Kalcioglu, Z. I.; Mahmoodian, R.; Hu, Y.; Suo, Z.; Van Vliet, K. J. From Macro- to Microscale Poroelastic Characterization of Polymeric Hydrogels via Indentation. Soft Matter 2012, 8 (12), 3393-3398.

(41) Delavoipière, J.; Tran, Y.; Verneuil, E.; Heurtefeu, B.; Hui, C. Y.; Chateauminois, A. Friction of Poroelastic Contacts with Thin Hydrogel Films. Langmuir 2018, 34 (33), 96179626.

(42) Keidel, R.; Ghavami, A.; Lugo, D. M.; Lotze, G.; Virtanen, O.; Beumers, P.; Pedersen, J. S.; Bardow, A.; Winkler, R. G.; Richtering, W. Time-Resolved Structural Evolution during the Collapse of Responsive Hydrogels: The Microgel-to-Particle Transition. Sci. Adv. 2018, $4(4), 1-9$. 
(43) Hooke, C. J.; O’Donoghue, J. P. Elastohydrodynamic Lubrication of Soft, Highly Deformed Contacts. J. Mech. Eng. Sci. 2007, 14 (1), 34-48.

(44) Rubinstein, M.; Colby, R. H. Polymer Physics, 1st Ed.; Oxford University Press: New York, 2003.

(45) Voudouris, P.; Florea, D.; Van Der Schoot, P.; Wyss, H. M. Micromechanics of Temperature Sensitive Microgels: Dip in the Poisson Ratio near the LCST. Soft Matter 2013, 9 (29), 7158-7166.

(46) Stieger, M.; Richtering, W.; Pedersen, J. S.; Lindner, P. Small-Angle Neutron Scattering Study of Structural Changes in Temperature Sensitive Microgel Colloids. J. Chem. Phys. 2004, 120 (13), 6197-6206.

(47) Conley, G. M.; Aebischer, P.; Nöjd, S.; Schurtenberger, P.; Scheffold, F. Jamming and Overpacking Fuzzy Microgels: Deformation, Interpenetration, and Compression. Sci. Adv. 2017, 3 (10), 1-8. 